\title{
Estimation of Lower Bounded Scale Parameter of Rescaled F-distribution under Entropy Loss Function
}

\author{
N. Nematollahi ${ }^{\dagger, *}$ and M. Naser Esfahani ${ }^{\ddagger}$ \\ $\dagger$ Allameh Tabataba'i University \\ ‡ Islamic Azad University, Science and Research Branch
}

\begin{abstract}
We consider the problem of estimating the scale parameter $\beta$ of a rescaled F-distribution when $\beta$ has a lower bounded constraint of the form $\beta \geqslant a$, under the entropy loss function. An admissible minimax estimator of the scale parameter $\beta$, which is the pointwise limit of a sequence of Bayes estimators, is given. Also in the class of truncated linear estimators, the admissible estimators and the only minimax estimator of $\beta$ are obtained.
\end{abstract}

Keywords. Admissibility; entropy loss function; F-distribution; minimax estimation; restricted parameter space.

\section{Introduction}

Historically, much of the work on estimating the parameter of a distribution dealt with unconstrained parameter spaces. However, in many statistical problems there exists bounds on the values that unknown parameters can take. In such problems, usual estimators for the unconstrained parameter are neither admissible nor minimax when the parameter is restricted. The problem of estimation in restricted parameter spaces was first studied by Brunk (1955) and van Eeden (1957) and then grew rapidly. A large body of work concerned with estimation problems in restricted parameter spaces has reviewed by Marchand and Strawderman (2004) and van Eeden (2006).

* Corresponding author 
Let $X$ be a random variable with probability density function (pdf)

$$
f(x \mid \beta)=c(m, \alpha) \frac{\beta^{\alpha-1} x^{m-1}}{(\beta+x)^{\alpha+m-1}}=\frac{1}{\beta} c(m, \alpha) \frac{\left(\frac{x}{\beta}\right)^{m-1}}{\left(1+\frac{x}{\beta}\right)^{\alpha+m-1}}, \quad x>0,
$$

where $c(m, \alpha)=\frac{\Gamma(\alpha+m-1)}{\Gamma(m) \Gamma(\alpha-1)}, m>0, \alpha>1$ and $\beta>0$. Note that $\frac{X}{\beta}$ is distributed as the ratio of two independent gamma random variables. According to van Eeden and Zideck (1994 a, b), we refer to the distribution with density given in (1) as a rescaled $F$-distribution.

The problem of estimation of scale parameter $\beta$ in rescaled $F$-distribution (1) when $\beta$ has a lower bounded constraint of the form $\beta \geqslant a$ for some known $a>0$, was first studied by van Eeden and Zideck (1994 a, b). They consider, among many other estimation problems, the admissible and minimax estimation of $\beta(\geqslant a)$. They derived the admissible estimators and the only minimax estimator in the class of truncated linear estimators of the form

$$
C=\left\{\delta_{\rho} \mid \delta_{\rho}(X)=\max (a, \rho X), \quad a>0, \rho>0\right\},
$$

under the Squared Error Loss (SEL) function $(\delta-\beta)^{2}$ and Scale Invariant SEL (SISEL) function $\left(\frac{\delta}{\beta}-1\right)^{2}$. van Eeden (2000) showed that the only minimax estimator in the class $\mathrm{C}$, is minimax among all estimators.

Now consider the estimation of scale parameter $\beta$ under the entropy loss function

$$
L(\beta, \delta)=\frac{\delta}{\beta}-\ln \frac{\delta}{\beta}-1,
$$

which is known as Stein's loss. This loss is convex in $\delta$ and is not symmetric and it penalizes heavily under estimation. In estimation problems that overestimation is more serious than under-estimation, the entropy loss (3) is more appropriate than SEL and SISEL functions which are symmetric about the parameter value. Under the loss (3), it is easy to show that the best scale invariant estimator of $\beta>0$ in model (1) is $\delta_{0}(X)=\frac{\alpha-2}{m} X$, when $\alpha>2$.

Under the entropy loss function (3) and for general scale family of distributions, Kubokawa (2004) showed that the unrestricted Minimum Risk Equivariant (MRE) estimator $\delta_{0}(X)$ of scale parameter $\beta$ is minimax when $\beta \geqslant a$, and also the Generalized Bayes Estimator (GBE) of $\beta$ with respect to (w.r.t.) improper prior $\pi(\beta)=\frac{1}{\beta}, \beta \geqslant a$, dominates $\delta_{0}(X)$ and hence is minimax. Also Marchand and Strawderman (2005) extend the results of Kubokawa (2004) to a general class of convex loss functions and obtained 
class of dominating estimator of $\delta_{0}(X)$. The results of these authors do not adress the general and interesting question of admissibility of GBE of $\beta(\geqslant a)$, see Section 5 of Marchand and Strawderman (2005). The admissibility of GBE of lower-bounded scale parameter has been established for some special distributions and under SEL and SISEL functions (see for example Farell, 1964, van Eeden, 1995 and Jafari Jozani et al., 2002).

In this paper admissible and minimax estimation of scale parameter $\beta$, when $\beta \geqslant a$, in model (1) and under the loss (3) is considered. To this end, in Section 2, the admissibility of GBE of $\beta$, which is the pointwise limit of a sequence of Bayes estimators, is shown. In Section 3, the admissible estimators and the only minimax estimator in the class of truncated linear estimators (2) are obtained. Finally a conclusion is given in Section 4.

\section{An Admissible Minimax Estimator}

Let $X$ has pdf (1) with known $m>0, \alpha>1$ and unknown $\beta \geqslant a$. In this section, we find GBE of $\beta$ w.r.t. improper prior

$$
\pi(\beta)=\frac{1}{\beta}, \quad \beta \geqslant a
$$

and show that the GBE is admissible and minimax estimator of $\beta(\geqslant a)$ under the entropy loss function (3).

Similar to van Eeden (1995), consider the following sequence of proper prior density for $\beta$,

$$
\pi_{n}(\beta)=\frac{a^{\frac{1}{n}}}{n \beta^{1+\frac{1}{n}}}, \quad \beta \geqslant a, a>0, n=1,2, \ldots
$$

Then from (1) and (5), the posterior distribution of $\beta$ given $X=x$ is given by

$$
\begin{aligned}
\pi_{n}(\beta \mid x)= & \frac{\frac{1}{\beta^{\frac{1}{n}+2}} \cdot \frac{\left(\frac{x}{\beta}\right)^{m-1}}{\left(1+\frac{x}{\beta}\right)^{m+\alpha-1}}}{\int_{a}^{\infty} \frac{1}{\beta^{\frac{1}{n}+2}} \cdot \frac{\left(\frac{x}{\beta}\right)^{m-1}}{\left(1+\frac{x}{\beta}\right)^{m+\alpha-1}} d \beta} \\
= & \frac{\frac{\left(\frac{x}{\beta}\right)^{m+\frac{1}{n}+1}}{\left(1+\frac{x}{\beta}\right)^{m+\alpha-1}}}{\int_{a}^{\infty} \frac{\left(\frac{x}{\beta}\right)^{m+\frac{1}{n}+1}}{\left(1+\frac{x}{\beta}\right)^{m+\alpha-1}} d \beta}, \quad \beta \geqslant a, a>0, n=1,2, \ldots
\end{aligned}
$$


The Bayes estimator of $\beta$ under the loss (3) is $\delta^{\pi_{n}}(x)=\left\{E\left(\frac{1}{\beta} \mid x\right)\right\}^{-1}$, and from (6) we have

$$
\delta^{\pi_{n}}(x)=x \frac{\int_{a}^{\infty} \frac{\left(\frac{x}{\beta}\right)^{m+\frac{1}{n}+1}}{\left(1+\frac{x}{\beta}\right)^{m+\alpha-1}} d \beta}{\int_{a}^{\infty} \frac{\left(\frac{x}{\beta}\right)^{m+\frac{1}{n}+2}}{\left(1+\frac{x}{\beta}\right)^{m+\alpha-1}} d \beta}=x \frac{\int_{0}^{\frac{x}{a}} \frac{t^{m+\frac{1}{n}-1}}{(1+t)^{m+\alpha-1}} d t}{\int_{0}^{\frac{x}{a}} \frac{t^{m+\frac{1}{n}}}{(1+t)^{m+\alpha-1}} d t} .
$$

For $b>0, c>1$ and $y>0$, define

$$
g_{b, c}(y)=\frac{y^{b}}{(1+y)^{b+c-1}} \quad \text { and } \quad G_{b, c}(y)=\int_{0}^{y} \frac{t^{b}}{(1+t)^{b+c-1}} d t .
$$

Then from (8) we have

$$
\delta^{\pi_{n}}(x)=x \frac{G_{m_{n}-1, \alpha_{n}+1}\left(\frac{x}{a}\right)}{G_{m_{n}, \alpha_{n}}\left(\frac{x}{a}\right)},
$$

where $m_{n}=m+\frac{1}{n}$ and $\alpha_{n}=\alpha-\frac{1}{n}$. Using integration by part in integral $G_{b, c}(y)$ with $u=\left(\frac{t}{1+t}\right)^{b}$ and $d v=\frac{1}{(1+t)^{c-1}} d t$, for $c>2$ we have

$$
G_{b, c}(y)=-\frac{1}{c-2} g_{b, c-1}(y)+\frac{b}{c-2} G_{b-1, c+1}(y),
$$

so,

$$
\frac{G_{b-1, c+1}(y)}{G_{b, c}(y)}=\frac{c-2}{b}\left\{1+\frac{g_{b, c-1}(y)}{(c-2) G_{b, c}(y)}\right\} .
$$

From (9) and (10), the Bayes estimator of $\beta$ for $\alpha>3$ is given by

$$
\delta^{\pi_{n}}(x)=\frac{\left(\alpha_{n}-2\right) x}{m_{n}}\left\{1+\frac{g_{m_{n}, \alpha_{n}-1\left(\frac{x}{a}\right)}}{\left(\alpha_{n}-2\right) G_{m_{n}, \alpha_{n}}\left(\frac{x}{a}\right)}\right\}
$$

and

$$
\begin{aligned}
\lim _{n \rightarrow \infty} \delta^{\pi_{n}}(x) & =\frac{(\alpha-2) x}{m}\left\{1+\frac{g_{m, \alpha-1\left(\frac{x}{a}\right)}}{(\alpha-2) G_{m, \alpha}\left(\frac{x}{a}\right)}\right\} \\
& =\delta^{\pi}(x) \quad \text { (say). }
\end{aligned}
$$

Note that $\delta^{\pi}(X)$ is the generalized Bayes (and limiting Bayes) estimator of $\beta(\geqslant a)$ w.r.t. improper prior (4) under the loss (3). In the following theorem we show that the GBE $\delta^{\pi}(X)$ is an admissible estimator of $\beta(\geqslant a)$ under the loss (3). We use Blyth's (1951) method for proof of admissibility. 
Theorem 1. Let $X$ has pdf (1) with $m>1, \alpha \geqslant 4$ and $\beta \geqslant a$. Then under the loss (3), the GBE given in (12) is an admissible estimator of $\beta$.

Proof. The loss function (3) satisfy $L(\beta, \delta)=L\left(\frac{\beta}{a}, \frac{\delta}{a}\right)$. So, without loss of generality we can take $a=1$. Since the risk function of an estimator $\delta$ under the loss (3) is continuous in $\beta$, we can use Blyth's (1951) method. So, we must show that for all $\eta>0$ such that $\theta-\eta \geqslant a$,

$$
\lim _{n \rightarrow \infty} \frac{n\left\{\Pi_{n}(\beta+\eta)-\Pi_{n}(\beta-\eta)\right\}}{n\left\{r\left(\pi_{n}, \delta^{\pi}\right)-r\left(\pi_{n}, \delta^{\pi_{n}}\right)\right\}}=+\infty,
$$

where $\Pi_{n}$ is the distribution function of $\beta$ with $\operatorname{pdf}(5), \delta^{\pi_{n}}$ is Bayes estimator of $\beta$ w.r.t. prior (5) and $r\left(\pi_{n}, \delta^{\pi}\right), r\left(\pi_{n}, \delta^{\pi_{n}}\right)$ are the Bayes risks of $\delta^{\pi}$ and $\delta^{\pi_{n}}$ w.r.t to the prior $\pi_{n}$, respectively. From (5) and using L'Hopital's rule we have

$$
\begin{aligned}
\lim _{n \rightarrow \infty} n\left\{\Pi_{n}(\beta+\eta)-\Pi_{n}(\beta-\eta)\right\} & =\lim _{n \rightarrow \infty} \int_{\beta-\eta}^{\beta+\eta} \frac{1}{t^{1+\frac{1}{n}}} d t \\
& =\lim _{n \rightarrow \infty} \frac{e^{-\frac{1}{n} \ln (\beta-\eta)}-e^{-\frac{1}{n} \ln (\beta+\eta)}}{\frac{1}{n}} \\
& =\ln \frac{\beta+\eta}{\beta-\eta}>0 .
\end{aligned}
$$

Also from (11) and (12) with $a=1$, we have

$$
\begin{aligned}
n\left\{r\left(\pi_{n}, \delta^{\pi}\right)-r\left(\pi_{n}, \delta^{\pi_{n}}\right)\right\}= & \left(\frac{\alpha-2}{m}-\frac{\alpha_{n}-2}{m_{n}}\right) \int_{1}^{\infty} \int_{0}^{\infty} \frac{x}{\beta^{\frac{1}{n}+2}} f(x \mid \beta) d x d \beta \\
+ & \int_{1}^{\infty} \int_{0}^{\infty}\left\{\frac{x}{m \beta} \cdot \frac{g_{m, \alpha-1}(x)}{G_{m, \alpha}(x)}-\frac{x}{m_{n} \beta} \cdot \frac{g_{m_{n}, \alpha_{n}-1}(x)}{G_{m_{n}, \alpha_{n}}(x)}\right\} \\
\times & \frac{1}{\beta^{\frac{1}{n}+1}} f(x \mid \beta) d x d \beta \\
+ & \left\{\ln \frac{\left(\alpha_{n}-2\right) m}{(\alpha-2) m_{n}}\right\} \int_{1}^{\infty} \int_{0}^{\infty} \frac{1}{\beta^{\frac{1}{n}+1}} f(x \mid \beta) d x d \beta \\
+ & \int_{1}^{\infty} \int_{0}^{\infty}\left[\ln \left\{1+\frac{g_{m_{n}, \alpha_{n}-1}(x)}{\left(\alpha_{n}-2\right) G_{m_{n}, \alpha_{n}}(x)}\right\}\right. \\
& \left.-\ln \left\{1+\frac{g_{m, \alpha-1}(x)}{(\alpha-2) G_{m, \alpha}(x)}\right\}\right] \frac{1}{\beta^{\frac{1}{n}+1}} f(x \mid \beta) d x d \beta \\
= & J_{1 n}+J_{2 n}+J_{3 n}+J_{4 n} .
\end{aligned}
$$


Note that

$$
\begin{aligned}
& J_{1 n}=\left(\frac{\alpha-2}{m}-\frac{\alpha_{n}-2}{m_{n}}\right) \frac{m}{\alpha-2} \int_{1}^{\infty} \beta \frac{1}{\beta^{\frac{1}{n}+2}} d \beta=n\left\{1-\frac{m\left(\alpha_{n}-2\right)}{m_{n}(\alpha-2)}\right\}, \\
& J_{3 n}=\left\{\ln \frac{m\left(\alpha_{n}-2\right)}{m_{n}(\alpha-2)}\right\} \int_{1}^{\infty} \frac{1}{\beta^{\frac{1}{n}+1}} d \beta=n\left\{\ln \frac{m\left(\alpha_{n}-2\right)}{m_{n}(\alpha-2)}\right\} .
\end{aligned}
$$

So, using L'Hopital's rule we have

$$
\begin{aligned}
& \lim _{n \rightarrow \infty} J_{1 n}=\lim _{n \rightarrow \infty} \frac{1-\frac{m\left(\alpha-\frac{1}{n}-2\right)}{\left(m+\frac{1}{n}\right)(\alpha-2)}}{\frac{1}{n}}=\frac{m+\alpha-2}{m(\alpha-2)} \\
& \lim _{n \rightarrow \infty} J_{3 n}=\lim _{n \rightarrow \infty} \frac{\ln \left\{m\left(\alpha-\frac{1}{n}-2\right)\right\}-\ln \left\{\left(m+\frac{1}{n}\right)(\alpha-2)\right\}}{\frac{1}{n}}=-\frac{m+\alpha-2}{m(\alpha-2)} .
\end{aligned}
$$

Hence $\lim _{m \rightarrow \infty}\left(J_{1 m}+J_{3 m}\right)=0$. Using the transformation $t=\frac{u}{1-u}$ in integral of $G_{b, c}(x)$ in (8), for $c \geqslant 3$ we have

$$
\begin{aligned}
G_{b, c}(x) & =\int_{0}^{x} \frac{t^{b}}{(1+t)^{b+c-1}} d t \\
& =\int_{0}^{\frac{x}{1+x}} u^{b}(1-u)^{c-3} d u \geqslant\left(\frac{1}{1+x}\right)^{c-3} \int_{0}^{\frac{x}{1+x}} u^{b} d u \\
& =\frac{x^{b+1}}{(b+1)(1+x)^{b+c-2}},
\end{aligned}
$$

therefore

$$
\frac{g_{b, c-1}(x)}{G_{b, c}(x)}<\frac{b+1}{x}
$$

Now for $\alpha \geqslant 4$, i.e., $\alpha_{n}-3 \geqslant 1-\frac{1}{n} \geqslant 0$, from (16) and $\ln (x+1) \leqslant x, x>0$, we have

$$
\begin{aligned}
\left|\left\{\frac{x}{m \beta} \cdot \frac{g_{m, \alpha-1}(x)}{G_{m, \alpha}(x)}-\frac{x}{m_{n} \beta} \cdot \frac{g_{m_{n}, \alpha_{n}-1}(x)}{G_{m_{n}, \alpha_{n}}(x)}\right\} \frac{1}{\beta^{\frac{1}{n}+1}}\right| & \leqslant\left(\frac{m+1}{m}+\frac{m_{n}+1}{m_{n}}\right) \frac{1}{\beta^{\frac{1}{n}+2}} \\
& \leqslant \frac{2(m+1)}{m} \cdot \frac{1}{\beta^{2}}
\end{aligned}
$$


and

$$
\begin{gathered}
\left|\left[\ln \left\{1+\frac{g_{m_{n}, \alpha_{n}-1}(x)}{\left(\alpha_{n}-2\right) G_{m_{n}, \alpha_{n}}(x)}\right\}-\ln \left\{1+\frac{g_{m, \alpha-1}(x)}{(\alpha-2) G_{m, \alpha}(x)}\right\}\right] \frac{1}{\beta^{\frac{1}{n}+1}}\right| \\
\leqslant\left\{\frac{g_{m_{n}, \alpha_{n}-1}(x)}{\left(\alpha_{n}-2\right) G_{m_{n}, \alpha_{n}}(x)}+\frac{g_{m, \alpha-1}(x)}{(\alpha-2) G_{m, \alpha}(x)}\right\} \frac{1}{\beta^{\frac{1}{n}+1}} \\
\leqslant\left(\frac{m_{n}+1}{\alpha_{n}-2}+\frac{m+1}{\alpha-2}\right) \frac{1}{\beta x} \leqslant\left(\frac{m+2}{\alpha-3}+\frac{m+1}{\alpha-2}\right) \frac{1}{\beta x} .
\end{gathered}
$$

Since $E\left(\frac{1}{X}\right)=\frac{\alpha-1}{\beta(m-1)}, m>1$ and for $\alpha \geqslant 4$

$$
\int_{1}^{\infty} \int_{0}^{\infty} \frac{2(m+1)}{m} \cdot \frac{1}{\beta^{2}} f(x \mid \beta) d x d \beta=\frac{2(m+1)}{m}<\infty
$$

and

$$
\begin{aligned}
\int_{1}^{\infty} \int_{0}^{\infty}\left(\frac{m+2}{\alpha-3}+\frac{m+1}{\alpha-2}\right) \frac{1}{\beta x} f(x \mid \beta) & d x d \beta \\
& =\frac{\alpha-1}{m-1}\left(\frac{m+2}{\alpha-3}+\frac{m+1}{\alpha-2}\right)<\infty
\end{aligned}
$$

so from Lebesgue dominated convergence theorem, $\lim _{n \rightarrow \infty} J_{2 n}=\lim _{n \rightarrow \infty} J_{4 n}=0$, and hence from (15)

$$
\lim _{n \rightarrow \infty} n\left\{r\left(\pi_{n}, \delta^{\pi}\right)-r\left(\pi_{n}, \delta^{\pi_{n}}\right)\right\}=0 .
$$

From (14) and (17), we conclude (13), which completes the proof.

Remark 1. From Theorem 2.4 of Kubokawa (2004) and Corollary 4 of Marchand and Strawderman (2005), we can conclude that the GBE $\delta^{\pi}(x)$ in (12) is a minimax estimator of $\beta$ when $\beta \geqslant a$, and hence $\delta^{\pi}(x)$ is an admissible minimax estimator of $\beta$ under entropy loss (3).

\section{Admissibility and Minimaxity of Truncated lin- ear Estimators}

In this section we discuss the admissibility and minimaxity of truncated linear estimators in the class $\mathrm{C}$ given by (2). If $X$ be a random variable 
with pdf (1), then the MRE estimator of $\beta$ under entropy loss $(3)$ is $\delta_{0}(X)=$ $\frac{\alpha-2}{m} X$ when $\alpha>2$ and $\beta$ is unrestricted i.e., $\beta>0$. From Theorem 2.3 of kubokawa (2004) and Corollary 11 of Marchand and Strawderman (2005) we can conclude that the estimator $\delta_{0}(X)$ in restricted parameter space $\beta \geqslant a$ is minimax and any minimax estimator of $\beta$ has minimax risk equal to constant risk $\delta_{0}$. Since

$$
\begin{aligned}
\operatorname{Minimax} \text { Value } & =E_{\beta}\left\{\frac{(\alpha-2) X}{m \beta}-\ln \frac{(\alpha-2) X}{m \beta}-1\right\} \\
& =1-\eta(\alpha, m)-\ln \frac{\alpha-2}{m}-1 \\
& =\ln \frac{m}{\alpha-2}-\eta(\alpha, m),
\end{aligned}
$$

where $\eta(\alpha, m)=E_{\beta=1}[\ln X] \leqslant \ln E_{\beta=1}(X)=\ln \frac{m}{\alpha-2}$, so, it is easy to verify that a (necessary) and sufficient condition for an estimator $\delta_{M}$ to be minimax estimator of $\beta \geqslant a$ under the entropy loss function (3) is given by

$$
R\left(\beta, \delta_{M}\right) \leqslant \sup _{\beta \geqslant a} R\left(\beta, \delta_{M}\right) \leqslant \ln \frac{m}{\alpha-2}-\eta(\alpha, m) .
$$

From (18), or equivalently from Theorem 2.4 of Kubokawa (2004) and Remark 5 of Marchand and Strawderman (2005), the truncated version of $\delta_{0}(X)$, i.e., $\delta_{\frac{\alpha-2}{m}}(X)=\max \left(a, \frac{\alpha-2}{m} X\right)$, is minimax estimator of $\beta(\geqslant a)$ under the entropy loss function (3). The estimator $\delta_{\frac{\alpha-2}{m}}(X)$ belongs to the class $C$ of truncated linear estimators of $\beta(\geqslant a)$ which is given by (2). This class was studied by van Eeden and Zidek (1994 a, b) and van Eeden (1995) for the estimation of lower bounded scale parameter of rescaled $\mathrm{F}$ and gamma distributions, respectively, under SISEL function. We want to characterize the admissible and minimax estimators of $\beta(\geqslant a)$ in the class $C$ under entropy loss function (3). In the following theorem we show that exactly one estimator in the class $C$ is minimax estimator.

Theorem 2. Let $X$ be a random variable with $p d f(1)$ where $\beta \geqslant a$ and $\alpha>2$. Then under the loss (3), the estimator $\delta_{\frac{\alpha-2}{m}}(X)=\max \left(a, \frac{\alpha-2}{m} X\right)$ in the class $C$ is minimax estimator of $\beta(\geqslant a)$, and $\stackrel{m}{n o}$ other estimator in $C$ is minimax. 
Proof. From (18), it suffices to show that

$$
\begin{aligned}
& \sup _{\beta \geqslant a} R\left(\beta, \delta_{\frac{\alpha-2}{m}}\right)=\ln \frac{m}{\alpha-2}-\eta(\alpha, m) \\
& \sup _{\beta} R\left(\beta, \delta_{\rho}\right)>\ln \frac{m}{\alpha-2}-\eta(\alpha, m) \quad \text { for } \quad \rho \neq \frac{\alpha-2}{m} .
\end{aligned}
$$

The risk function of $\delta_{\rho}$ under the loss (3) is

$$
\begin{aligned}
R\left(\beta, \delta_{\rho}\right)= & E_{\beta}\left(\frac{\rho X}{\beta}-\ln \frac{\rho X}{\beta}-1\right) \\
& +\int_{0}^{\frac{a}{\rho \beta}}\left[\left(\frac{a}{\beta}-\ln \frac{a}{\beta}\right)-\{\rho s-\ln (\rho s)\}\right] f(s \mid 1) d s \\
= & \frac{\rho m}{\alpha-2}-\ln \rho-\eta(\alpha, m)-1+\left(\frac{a}{\beta}-\ln \frac{a}{\beta}\right) \int_{0}^{\frac{a}{\rho \beta}} f(s \mid 1) d s \\
& -\int_{0}^{\frac{a}{\rho \beta}}\{\rho s-\ln (\rho s)\} f(s \mid 1) d s .
\end{aligned}
$$

So, for all $\rho>0$ we have

$$
\frac{\partial}{\partial \beta} R\left(\beta, \delta_{\rho}\right)=\frac{\beta-a}{\beta^{2}} \int_{0}^{\frac{a}{\rho \beta}} f(s \mid 1) d s>0 .
$$

Hence $R\left(\beta, \delta_{\rho}\right)$ is a strictly increasing function of $\beta$. Therefore using L'Hospital's rule, we have

$$
\begin{aligned}
\sup _{\beta \geqslant a} R\left(\beta, \delta_{\rho}\right) & =\lim _{\beta \rightarrow \infty} R\left(\beta, \delta_{\rho}\right) \\
& =\frac{\rho m}{\alpha-2}-\ln \rho-\eta(\alpha, m)-1-\lim _{\beta \rightarrow \infty}\left\{\ln \left(\frac{a}{\beta}\right) \int_{0}^{\frac{a}{\rho \beta}} f(s \mid 1) d s\right\} \\
& =\frac{\rho m}{\alpha-2}-\ln \rho-\eta(\alpha, m)-1 \\
& =h_{\alpha, m}(\rho), \quad \text { (say). }
\end{aligned}
$$

Note that $h_{\alpha, m}(\rho)$ is a strictly convex function of $\rho$ and takes its minimum at $\rho=\frac{\alpha-2}{m}$. Therefore (19) holds true, which completes the proof.

Now consider the following subclass of $C$

$$
C^{\prime}=\left\{\delta_{\rho} \in c \mid 0<\rho \leqslant \frac{\alpha-2}{m}\right\} .
$$


We want to show that $C^{\prime}$ consist of all those estimators in $C$ which are admissible in $C$. To do this, we use the technique of van Eeden and Zideck (1994 b), which they used for SISEL function. For the proof of this result, the following lemmas are needed.

For convenience, let $p=m$ and $q=\alpha-1$ in the expression (1), and let $g(x, p, q)=\frac{x^{p}}{(1+x)^{p+q}}$ for $x>0$, then from (1) we have $f(x \mid 1)=c(p, q+$ 1) $g(x, p-1, q+1), x>0$.

Lemma 1. For $p>0$ and $q>0$,

$$
\frac{\partial}{\partial \rho} R\left(\beta, \delta_{\rho}\right) \lesseqgtr 0 \Longleftrightarrow \rho \int_{\frac{a}{\rho \beta}}^{\infty} g(y, p, q) d y \lesseqgtr \int_{\frac{a}{\rho \beta}}^{\infty} g(y, p-1, q+1) d y .
$$

Proof. Note that

$$
\begin{aligned}
R\left(\beta, \delta_{\rho}\right) & =\int_{0}^{\frac{a}{\rho}}\left(\frac{a}{\beta}-\ln \frac{a}{\beta}-1\right) f(x \mid \beta) d x+\int_{\frac{a}{\rho}}^{\infty}\left(\frac{\rho x}{\beta}-\ln \frac{\rho x}{\beta}-1\right) f(x \mid \beta) d x \\
& =\left(\frac{a}{\beta}-\ln \frac{a}{\beta}-1\right)+\int_{\frac{a}{\rho \beta}}^{\infty}\left\{(\rho y-\ln \rho y)-\left(\frac{a}{\beta}-\ln \frac{a}{\beta}\right)\right\} f(y \mid 1) d y .
\end{aligned}
$$

So,

$$
\frac{\partial}{\partial \rho} R\left(\beta, \delta_{\rho}\right)=\frac{a}{\rho^{2} \beta} \int_{\frac{a}{\rho \beta}}^{\infty}\left(y-\frac{1}{\rho}\right) f(y \mid 1) d y
$$

and hence

$$
\begin{aligned}
\frac{\partial}{\partial \rho} R\left(\beta, \delta_{\rho}\right) \lesseqgtr 0 & \Longleftrightarrow \int_{\frac{a}{\rho \beta}}^{\infty} y f(y \mid 1) d y \lesseqgtr \frac{1}{\rho} \int_{\frac{a}{\rho \beta}}^{\infty} f(y \mid 1) d y \\
& \Longleftrightarrow \rho \int_{\frac{a}{\rho \beta}}^{\infty} g(y, p, q) d y \lesseqgtr \int_{\frac{a}{\rho \beta}}^{\infty} g(y, p-1, q+1) d y
\end{aligned}
$$

which completes the proof.

Lemma 2. For $p>0$ and $q>1$, let

$$
H(k)=\frac{\int_{k}^{\infty} g(y, p-1, q+1) d y}{\int_{k}^{\infty} g(y, p, q) d y}, \quad k \geqslant 0,
$$

then $H(k)$ satisfy 
(i) $H(0)=\frac{q-1}{p}$

(ii) $\lim _{k \rightarrow \infty} H(k)=0$ and $\lim _{k \rightarrow \infty} k H(k)=\frac{q-1}{q}$

(iii) $H(k)$ is strictly decreasing and $k H(k)$ is strictly increasing in $k$.

The proof of Lemma 2 is similar to proof of Lemmas 4.2 and 4.3 of van Eeden and Zideck (1994 b), in which $p$ and $q$ are replaced by $p-1$ and $q+1$, respectively, and hence is omitted.

The properties of the risk function of the estimators in the class $C$ as a function of $\rho$ and $\beta$ are given in the following theorem.

Theorem 3. For $\alpha>2$ and $\beta \geqslant a>0$, there exists a $\rho(\beta)$ for every $\beta>\frac{a(\alpha-1)}{\alpha-2}$ such that

(i) $\frac{\partial}{\partial \rho} R\left(\beta, \delta_{\rho}\right) \lesseqgtr 0 \Longleftrightarrow \rho \lesseqgtr \rho(\beta)$,

(ii) $0<\rho(\beta)<\frac{\alpha-2}{m}$,

(iii) $\rho(\beta)$ is strictly increasing in $\beta$,

(iv) $\rho(\beta) \rightarrow 0$ as $\beta \rightarrow \frac{a(\alpha-1)}{\alpha-2}$ and $\rho(\beta) \rightarrow \frac{\alpha-2}{m}$ as $\beta \rightarrow \infty$,

(v) $R\left(\beta, \delta_{\rho}\right)$ is strictly increasing in $\rho$ for $a \leqslant \beta \leqslant \frac{a(\alpha-1)}{\alpha-2}$,

(vi) There exists $\beta(\rho)>\frac{a(\alpha-1)}{\alpha-2}$ such that $R\left(\beta(\rho), \delta_{\frac{\alpha-2}{m}}\right)<R\left(\beta(\rho), \delta_{\rho}\right)$ for every $\rho \in\left(0, \frac{\alpha-2}{m}\right)$.

Proof. Using Lemmas 1 and 2, the proof of (i)-(v) is similar to the proof of Theorem 2.2 (i)-(iv) of van Eeden and Zideck (1994 b). For a proof of (vi), note that from (22) we have

$$
\begin{aligned}
R\left(\beta, \delta_{\frac{\alpha-2}{m}}\right)-R\left(\beta, \delta_{\rho}\right)= & \int_{\frac{a m}{(\alpha-2) \beta}}^{\infty}\left\{\left(\frac{\alpha-2}{m} y-\ln \frac{\alpha-2}{m} y\right)\right. \\
& \left.-\left(\frac{a}{\beta}-\ln \frac{a}{\beta}\right)\right\} f(y \mid 1) d y \\
& -\int_{\frac{a}{\rho \beta}}^{\infty}\left\{(\rho y-\ln \rho y)-\left(\frac{a}{\beta}-\ln \frac{a}{\beta}\right)\right\} f(y \mid 1) d y .
\end{aligned}
$$


Now it is sufficient to prove that the limit of right hand side of (23) when $\beta \rightarrow \infty$ is negative. But this limit is

$$
\begin{aligned}
\int_{0}^{\infty}\left\{\left(\frac{\alpha-2}{m} y-\ln \frac{\alpha-2}{m} y\right)-(\rho y-\ln \rho y)\right\} f(y \mid 1) d y= & 1-\frac{\rho m}{\alpha-2} \\
& +\ln \frac{\rho m}{\alpha-2}<0,
\end{aligned}
$$

since $\ln x<x-1, x>0$, which completes the proof.

In the following theorem we characterize the admissible estimators in class $C$.

Theorem 4. If $\beta \geqslant a>0$ and $\alpha>2$, then

(i) $\delta_{\rho}$ dominates $\delta_{\rho^{\prime}}$ for $\frac{\alpha-2}{m} \leqslant \rho<\rho^{\prime}$,

(ii) Each estimator in the class $C^{\prime}$ given by (22) is admissible in the class $C$ given by (2).

The proof of Theorem 4 is similar to the proof of Theorem 2.3 of van Eeden and Zideck (1994 b) by replacing $\alpha, \mathrm{n}$ and $\hat{\beta}$ with $\alpha+1, m-1$ and $\delta$, respectively, and hence is omitted.

Remark 2. From Theorems 2 and 4 we conclude that the truncated linear estimators $\delta_{\rho}=\max (a, \rho X)$ of $\beta(\geqslant a)$ in the class $C$ are admissible if and only if $0<\rho \leqslant \frac{\alpha-2}{m}$ and the only admissible and minimax estimator in this class is $\frac{\alpha-2}{m}(X)$, which is the truncated version of MRE estimator $\delta_{0}(X)=$ $\frac{\alpha-2}{m} X$.

Remark 3. Let $T$ and $W$ be two independent random variables with $\operatorname{Gamma}\left(m, \lambda_{1}\right)$ and $\operatorname{Gamma}\left(\alpha-1, \lambda_{2}\right)$ distributions , respectively. Then $X=\frac{T}{W}$ has rescaled F-distribution with pdf (1) where $\beta=\frac{\lambda_{1}}{\lambda_{2}}$. The distribution of the ratio of random variables are of interest in different problems such as biological and physical sciences, econometrics, classification, and ranking and selection. Examples of the use of the ratio of random variables include Mendelian inheritance ratios in genetics, mass to energy ratios in nuclear physics, target to control precipitation in meteorology, and inventory ratios in economics (Masoom Ali et al., 2007). The distribution of ratio 
of independent gamma random variables have some applications in statistical inference. An application of this distribution is given in the following example. For more details see Provost (1989) and Bowman et al. (1998).

Example 1. Let $X_{i 1}, \ldots, X_{i n_{i}}, i=1,2$ be two independent random samples from $\mathrm{N}\left(\mu_{i}, \sigma_{i}^{2}\right)$-distribution, $i=1,2$. Then $X=\frac{S_{1}^{2}}{S_{2}^{2}}$ has a rescaled F-distribution with pdf (1) where $\beta=\frac{\sigma_{1}^{2}}{\sigma_{2}^{2}}, m=\frac{n_{1}-1}{2}$ and $\alpha-1=\frac{n_{2}-1}{2}$. Now if we have a prior information $\sigma_{2}^{2} \leqslant \sigma_{1}^{2}$, then $\beta \geqslant 1$. So, to estimate the ratio of two normal variances $\beta=\frac{\sigma_{1}^{2}}{\sigma_{2}^{2}}$ with the restriction $\sigma_{2}^{2} \leqslant \sigma_{1}^{2}$, we can use the results of Sections 2 and 3 to find admissible and minimax estimators of $\beta(\geqslant 1)$.

\section{Conclusion}

In previous sections in estimation of scale parameter of rescaled F-distribution with pdf (1), we derive admissible and minimax estimators of scale parameter $\beta$ under entropy loss function (3), when $\beta$ has a lower bounded constraint of the form $\beta \geqslant a$. An admissible minimax estimator of $\beta(\geqslant a)$, which is the pointwise limit of a sequence of Bayes estimators, is given. In the class of truncated linear estimators $(2)$ of $\beta(\geqslant a)$, we derive the only minimax estimator and characterize the admissible estimators in this class.

It is worth to consider estimation of lower bounded scale parameter of rescaled F-distribution under other scale invariant loss functions, and we are working on it for driving further nice results.

\section{Acknowledgment}

The authors are grateful to the editor, and two anonymous referees for making helpful comments and suggestions on an earlier version of this article. Research of the first author was supported by the research council of Allameh Tabataba'i University.

\section{References}

Blyth, C.R. (1951). On minimax statistical procedures and their admissibility. Ann. Math. Statist., 22, 22-42. 
Bowman, K.O., Shenton, L.R. and Gailey, P.C. (1998). Distribution of the ratio of gamma variates. Commun. Statist. Simulation and Computation, 27, 1-19.

Brunk, H.D. (1955). Maximum likelihood estimates of monotone parameters. Ann. Math. Statist., 26, 607-616.

Farrell, R.H. (1964). Estimators of a location parameter in the absolutely continuous case. Ann. Math. Statist., 35, 949-998.

Jafari Jozani, M., Nematollahi, N. and Shafie, K. (2002). An admissible minimax estimator of a bounded scale-parameter in a subclass of the exponential family under scale-invariant squared-error loss. Statist. Probab. Lett., 60, 437-444.

Kubokawa, T. (2004). Minimaxity in estimation of restricted parameters. J. Japan Statist. Soc., 34(2), 1-19.

Marchand, E. and Strawderman, W.E. (2004). Improving on the minimum risk equivariant estimator of a location parameter which is constrained to an interval or a half-interval. Ann. Inst. Statist. Math., 16, 155-160.

Marchand, E. and Strawderman, W.E. (2005). On improving on the minimax risk equivariant estimator of scale parameter under a lower-bounded constraint. J. Statist. Plann. Infer., 134, 90-101.

Masoom Ali, M., Pal, M. and Woo, J. (2007). On the ratio of inverted gamma variates. Austrian J. Statist. 36(2), 153-159.

Provost, S. B. (1989). On the distribution of the ratio of powers of sums of gamma random variables. Pakistan J. Statist., 5, 157-174.

van Eeden, C. (1957). Maximum likelihood estimation of partially or completely ordered parameters. Proc. Kon. Ned. Akad. V. Wet. 60 A, 128-136, 201-211.

van Eeden, C. (1995). Minimax estimation of a lower bounded scale-parameter of a gamma distribution for scale-invariant squared error loss. Canad. J. Statist. 23, 245-256.

van Eeden, C. (2000). Minimax estimation of a lower-bounded scale-parameter of an Fdistribution. Statist. Probab. Lett. 46, 283-286.

van Eeden, C. (2006). Restricted parameter space estimation problems, admissibility and minimaxity properties, Springer, Lecture notes in statistics, $\mathbf{1 8 8 .}$

van Eeden, C. and Zidek, J.V. (1994 a). Group Bayes estimation of the exponential mean: A retrospective of the Wald theory. In Statistical Decision Theory and Related Topics V (S.S., Gupta and J.O. Berger, eds.), 35-49. Springer-verlag.

van Eeden, C. and Zidek, J.V. (1994 b). Group Bayes estimation of the exponential mean: A preposterior analysis. Test 3, 125-143; corrections p. 247. 
N. Nematollahi

Department of Statistics,

Allameh Tabataba'i University,

Tehran, Iran.

email:nematollahi@atu.ac.ir

\section{Naser Esfahani}

Department of Statistics,

Science and Research Branch,

Islamic Azad University,

Tehran, Iran.

email: nasresfahani@iaun.ac.ir 